Lindsay I. Berrigan, $\mathrm{PhD}$

John D. Fisk, PhD

Scott B. Patten, MD,

$\mathrm{PhD}$

Helen Tremlett, PhD

Christina Wolfson, PhD

Sharon Warren, PhD

Kirsten M. Fiest, $\mathrm{PhD}$

Kyla A. McKay, BSc

Ruth Ann Marrie, MD,

$\mathrm{PhD}$

For the CIHR Team in the Epidemiology and Impact of Comorbidity on Multiple Sclerosis (ECoMS)

Correspondence to

Dr. Berrigan:

lberriga@stfx.ca
Supplemental data at Neurology.org

\section{Health-related quality of life in multiple sclerosis}

\author{
Direct and indirect effects of comorbidity
}

\section{ABSTRACT}

Objective: To evaluate the direct and indirect influences of physical comorbidity, symptoms of depression and anxiety, fatigue, and disability on health-related quality of life (HRQoL) in persons with multiple sclerosis (MS).

Methods: A large ( $n=949$ ) sample of adults with MS was recruited from 4 Canadian MS clinics. HRQoL was assessed using the patient-reported Health Utilities Index Mark 3. Expanded Disability Status Scale scores, physical comorbidity, depression, anxiety, and fatigue were evaluated as predictors of $\mathrm{HRQOL}$ in a cross-sectional path analysis.

Results: All predictors were significantly associated with HRQoL and together accounted for a large proportion of variance (63\%). Overall, disability status most strongly affected HRQoL $(\beta=-0.52)$ but it was closely followed by depressive symptoms $(\beta=-0.50)$. The direct associations of physical comorbidity and anxiety with HRQoL were small $(\beta=-0.08$ and -0.10 , respectively), but these associations were stronger when indirect effects through other variables (depression, fatigue) were also considered (physical comorbidity: $\beta=-0.20$; anxiety: $\beta=-0.34$ ).

Conclusions: Increased disability, depression and anxiety symptoms, fatigue, and physical comorbidity are associated with decreased HRQoL in MS. Disability most strongly diminishes HRQoL and, thus, interventions that reduce disability are expected to yield the most substantial improvement in HRQoL. Yet, interventions targeting other factors amenable to change, particularly depression but also anxiety, fatigue, and physical comorbidities, may all result in meaningful improvements in $\mathrm{HRQ} \mathrm{L}$, as well. Our findings point to the importance of further research confirming the efficacy of such interventions. Neurology ${ }^{\circledR} 2016 ; 86: 1417-1424$

\section{GLOSSARY}

CFI = Comparative Fit Index; $\mathbf{C l}=$ confidence interval; $\mathbf{D S M}-\mathbf{I V}=$ Diagnostic and Statistical Manual of Mental Disorders, 4th edition; EDSS = Expanded Disability Status Scale; HADS = Hospital Anxiety and Depression Scale; HADS-A = Hospital Anxiety and Depression Scale anxiety subscale; HADS-D = Hospital Anxiety and Depression Scale depression subscale; HRQoL = health-related quality of life; HUI3 = Health Utilities Index Mark 3; MS = multiple sclerosis; NARCOMS = North American Research Committee on Multiple Sclerosis; RMSEA = root mean square error of approximation.

Persons with multiple sclerosis (MS) report lower health-related quality of life (HRQoL) as compared to general and other chronic disease populations. ${ }^{1-3}$ Factors such as age, sex, socioeconomic status, disability status, depression, and fatigue have been associated with HRQoL in MS. ${ }^{4}$ Comorbidity is high in MS and the most prevalent comorbidities include mental health conditions, such as depression and anxiety, and physical conditions, such as hypertension, hyperlipidemia, and chronic lung disease. 5 While comorbidity is associated with reduced HRQoL for other chronic diseases, ${ }^{6}$ little is known about its influence in MS.

Our objective was to evaluate the extent to which physical comorbidity, depression, anxiety, and other factors previously associated with HRQoL in MS (disability, fatigue) $)^{7,8}$ exert both direct and indirect influences on HRQoL. For example, if increasing physical comorbidity

\footnotetext{
From the Department of Psychology (L.I.B.), St. Francis Xavier University, Antigonish; Department of Psychiatry (L.I.B., J.D.F.), Department of Medicine (J.D.F.), Dalhousie University, Halifax; Departments of Psychiatry and Community Health Sciences (S.B.P.), Cumming School of Medicine, University of Calgary; Division of Neurology, Faculty of Medicine (H.T., K.A.M.), University of British Columbia, Vancouver; Departments of Epidemiology \& Biostatistics, Occupational Health, \& Medicine (C.W.), McGill University, Montreal; Faculty of Rehabilitation Medicine (S.W.), University of Alberta, Edmonton; and Departments of Internal Medicine (K.M.F., R.A.M.) and Community Health Sciences (R. A.M.), College of Medicine, Faculty of Health Sciences, University of Manitoba, Winnipeg, Canada.

Coinvestigators are listed on the Neurology ${ }^{\circledR}$ Web site at Neurology.org.

Go to Neurology.org for full disclosures. Funding information and disclosures deemed relevant by the authors, if any, are provided at the end of the article.
} 
worsens fatigue and the increase in fatigue, in turn, reduces quality of life, this would represent an indirect effect of physical comorbidity on HRQoL. Previous research identifying factors that contribute to HRQoL in MS has primarily considered predictors independently. However, relations exist among them and their overlap may also represent mechanisms by which individual predictors further diminish HRQoL. Thus, we evaluated whether the predictors considered in the present study also affected HRQoL indirectly, by modifying one another, in addition to affecting HRQoL directly. We hypothesized that disability, physical comorbidity, and symptoms of anxiety and depression would have direct and indirect effects on HRQoL and that fatigue would have a direct influence on HRQoL.

METHODS Study population. From July 2010 through March 2011, 949 adults with definite MS were recruited through consecutive visits at $4 \mathrm{MS}$ clinics across Canada: Alberta, British Columbia, Manitoba, and Nova Scotia. See appendix e-1 on the Neurology ${ }^{\circledR}$ Web site at Neurology.org for sample size justification. Inclusion criteria were a confirmed diagnosis of definite MS according to the prevailing diagnostic criteria at the time, ${ }^{9,10}$ aged 18 years or older, fluent in English, residence in the province where data collection was occurring, and ability to provide informed consent.

Clinical information. We captured demographic and clinical information from medical records using a standardized data abstraction form including sex, date of birth, race, age at MS symptom onset, clinical course, and Expanded Disability Status Scale (EDSS) ${ }^{11}$ as recorded on the day of recruitment.

Self-report measures. HRQoL was operationalized as the functional effect of a health condition on physical and mental wellbeing from the perspective of the affected individual using continuous global utility scores from the Health Utilities Index Mark 3 (HUI3). The HUI3 is a 15 -item generic utility measure that assesses patient-reported health states with respect to vision, hearing, speech, mobility, dexterity, emotion, cognition, and pain. Attribute scores are aggregated into a single measure (global utility score) ranging from 0 (death) to 1 (perfect health) but allow for negative states considered worse than death. ${ }^{12}$ The multi-attribute scoring function used was derived from community preferences obtained for a random sample of Canadians. ${ }^{12}$ The HUI3 is valid and reliable in the MS population. ${ }^{13}$

Physical comorbidity was operationalized as the total number of physical illnesses other than MS ever diagnosed as reported on a validated comorbidity questionnaire. ${ }^{14}$ Although the questionnaire also asked about several psychiatric illnesses, these were not included in the physical comorbidity count. Instead, depression and anxiety, specifically, were represented in the analysis as severity of symptoms experienced in the past week based on continuous scores from the Hospital Anxiety and Depression Scale (HADS). The current level of symptoms was considered more relevant to perceived $\mathrm{HRQ}$ oL than having ever been diagnosed with anxiety or depression. The HADS is a 14-item selfadministered questionnaire for assessing symptoms of anxiety and depression in the medical outpatient clinic setting. ${ }^{15}$ In the MS population, a score of $\geq 8$ on the depression subscale (HADS-D) has a sensitivity of $90 \%$ and a specificity of $87.3 \%$ as compared to major depression defined by the Structured Clinical Interview for DSM-IV. ${ }^{16} \mathrm{~A}$ score of $\geq 8$ on the anxiety subscale (HADS-A) has a sensitivity of $88.5 \%$ and a specificity of $80.7 \%$ for generalized anxiety disorder. ${ }^{16}$

Fatigue was operationalized as the continuous total score on the Fatigue Impact Scale for Daily Use, a validated 8-item instrument. ${ }^{17}$ Each item is scored from 0 (no) to 4 (extreme problem), and the items are summed to achieve a total score.

Standard protocol approvals and patient consents. Institutional ethics approval was obtained at all sites and participants provided informed consent.

Statistical analysis. The relation between each of EDSS, physical comorbidity, depression, anxiety, fatigue, and HRQoL was evaluated using path analysis. Missing values were imputed using regression imputation and the models were estimated using maximum likelihood in Amos 20. ${ }^{18}$ Missing data were minimal: 5.0\% of EDSS, $2.0 \%$ of HADS-D, $1.8 \%$ of HADS-A, and $0.9 \%$ of HUI3 data were missing. Imputation was necessary because the technique used to test the significance of indirect effects (biascorrected bootstrap confidence intervals [CIs]) requires complete data. However, similar parameter estimates were obtained when either the original dataset or dataset with imputed values was analyzed.

Several indices were used to evaluate model fit. The $\chi^{2}$ test assesses the difference between the observed covariance matrix and the estimated covariance matrix under the restrictions imposed by the hypothesized model. ${ }^{19}$ Because trivial differences between sample and estimated covariance matrices are often significant with large samples such as ours, other fit indices were also considered. ${ }^{20}$ The Comparative Fit Index (CFI) assesses the proportionate improvement in fit by comparing the hypothesized model with the independence model (i.e., model with no correlations among observed variables). The root mean square error of approximation (RMSEA) estimates the discrepancy or lack of fit in the hypothesized model compared to the saturated model (i.e., model with correlations among all observed variables). A nonsignificant $(\alpha=0.05) \chi^{2}$ value, $C F I \geq 0.95$, and RMSEA $\leq 0.05$ indicate satisfactory model fit. ${ }^{19}$

Bias-corrected bootstrap CIs were used to estimate 95\% CIs for all model parameters. Parameters with CIs not including 0 were considered statistically significant at the 0.05 level. Tests of significance were based on unstandardized coefficients; however, standardized coefficients are presented and discussed to fully describe the results of the model. Please see the note below table 1 for direction on interpretation of path coefficients.

Figure 1 provides a simplified illustration of the model that was tested. The model included direct associations of the hypothesized predictors (EDSS, physical comorbidity, depression, anxiety, fatigue) and HRQoL. A correlation between physical comorbidity and EDSS was included, as were direct associations of both EDSS and physical comorbidity with depression, anxiety, and fatigue and both depression and anxiety with fatigue so that indirect effects through the latter variables could be examined. Because depression and anxiety frequently co-occur with no clear indication of temporal/causal priority, feedback effects were modeled between these 2 variables. ${ }^{21}$ The unstandardized coefficients of the bidirectional effects were restrained to be equal to allow for identification of the model. ${ }^{22}$ Finally, direct associations of 
Table 1 Path coefficients for the predictors of health-related quality of life (HRQoL) in the multiple sclerosis sample

\begin{tabular}{|c|c|c|c|c|c|c|c|c|c|c|c|c|}
\hline & \multicolumn{12}{|c|}{ Effects on HRQoL } \\
\hline $\begin{array}{l}\text { Disability status } \\
\text { (EDSS) }\end{array}$ & -0.06 & 0.004 & -0.068 to -0.053 & -0.40 & -0.02 & 0.003 & -0.025 to -0.013 & -0.12 & -0.08 & 0.004 & -0.087 to -0.070 & -0.52 \\
\hline Depression (HADS-D) & -0.03 & 0.003 & -0.031 to -0.019 & -0.28 & -0.02 & 0.002 & -0.023 to -0.016 & -0.22 & -0.04 & 0.003 & -0.049 to -0.039 & -0.50 \\
\hline Fatigue (DFIS) & -0.01 & 0.001 & -0.013 to -0.008 & -0.27 & - & - & - & - & -0.01 & 0.001 & -0.013 to -0.008 & -0.27 \\
\hline Physical comorbidities & -0.02 & 0.006 & -0.032 to -0.009 & -0.08 & -0.03 & 0.005 & -0.043 to -0.023 & -0.13 & -0.05 & 0.007 & -0.067 to -0.038 & -0.20 \\
\hline
\end{tabular}

Abbreviations: $\beta=$ standardized coefficient; $\mathrm{B}=$ unstandardized coefficient; $\mathrm{Cl}=$ confidence interval; DFIS = Daily Fatigue Impact Scale; EDSS = Expanded Disability Status Scale; HADS-A = Hospital Anxiety and Depression Scale-anxiety; HADS-D = Hospital Anxiety and Depression Scaledepression.

Larger values on the Health Utilities Index Mark 3 represent better HRQoL; therefore, negative coefficients should be interpreted as indicating worsening HRQoL. Standardized direct coefficients can be interpreted as the number of SD change in HRQoL that is associated with a 1-SD change in the predictor when all other predictors are held constant. Standardized indirect coefficients can be interpreted as the number of SD change in HRQoL when the predictor is held constant and the intermediate variable(s) changes by the amount it would have changed had the predictor increased by 1 SD unit. Total coefficients are the sum of direct and indirect coefficients. For example: HRQoL is expected to worsen by $0.40 \mathrm{SD}$ s for each 1-SD increase in disability status; HRQoL is expected to worsen by 0.12 SDs when depression, anxiety, and fatigue change by the amount associated with a 1-SD increase in disability status; via all direct and indirect influences presumed, HRQoL is expected to worsen by 0.52 SDs for each 1-SD increase in disability status.

important demographic/disease variables (sex, age, disease course, disease duration, disease-modifying therapy use) and HRQoL were included in the model so that the hypothesized predictors could be examined while accounting for these variables. Correlations between the hypothesized predictors and the demographic/disease variables were included where appropriate (all demographic/disease variables were correlated with one another as well as with the hypothesized predictors except for sex with age, sex with disease duration, and sex with fatigue). The demographic/disease variables and their associations are not shown in figure 1 to simplify interpretation.

Figure 1 Path analysis model of predictors of health-related quality of life (HRQoL) in multiple sclerosis (MS)

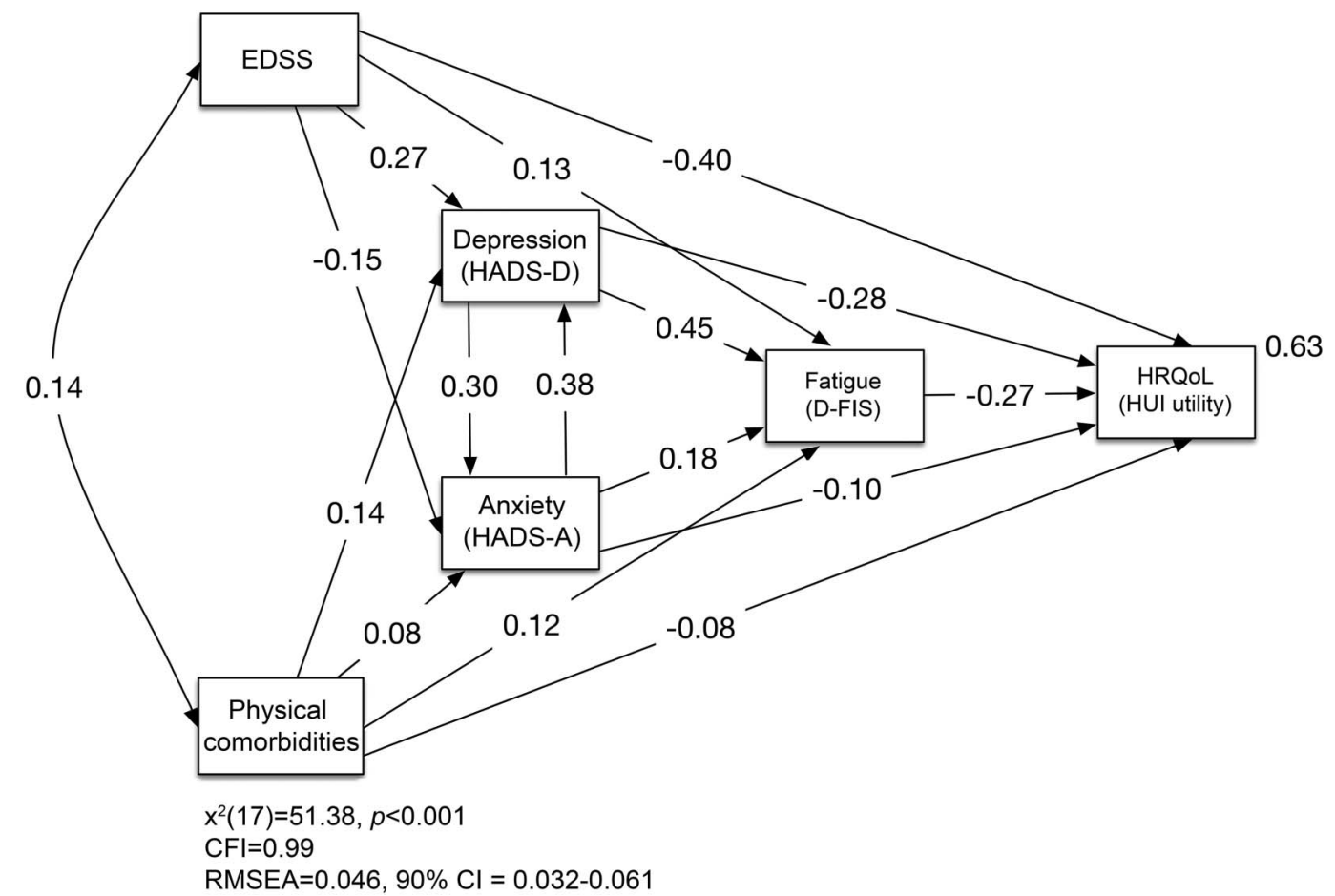

The value on the double-headed arrow is a correlation coefficient; values on single-headed arrows are standardized regression coefficients; the value to the upper right of the dependent variable is the squared multiple correlation. CFI = Comparative Fit Index; $\mathrm{Cl}$ = confidence interval; DFIS = Daily Fatigue Impact Scale; EDSS = Expanded Disability Status Scale; HADS-A = Hospital Anxiety and Depression Scale anxiety subscale; HADS-D = Hospital Anxiety and Depression Scale depression subscale; $\mathrm{HUI}=$ Health Utilities Index; RMSEA = root mean square error of approximation. 
RESULTS See figure 2 for the number of individuals at each stage of study. Participant characteristics and mean scores on the measures of interest are reported in table 2 . Of note, $63 \%$ of the sample had HRQoL scores that are considered to reflect severe disability (i.e., HUI3 global utility score $<0.70^{23}$ ). Thirty-nine percent of the sample reported clinically meaningful symptoms of anxiety and $21 \%$ reported clinically meaningful symptoms of depression (i.e., a score $\geq 8$ ). ${ }^{16}$ The number and percentage of participants reporting physical or psychiatric comorbidities is shown in table 3 . The most frequently reported comorbidities were depression (29.0\%), hypertension (17.8\%), migraine (17.3\%), hypercholesterolemia (12.4\%), and anxiety disorder (11.5\%).

Figure 1 presents the model fit results as well as standardized regression coefficients. All of the direct associations shown in figure 1 were statistically significant. EDSS and physical comorbidity were more strongly associated with depression $(\beta=0.27, \beta=$ 0.14 , respectively) than with anxiety $(\beta=-0.15$, $\beta=0.08$, respectively). Depression was most strongly associated with fatigue $(\beta=0.45)$, followed by anxiety $(\beta=0.18)$, and then EDSS and physical comorbidity, which had similar magnitudes of association with fatigue ( $\beta=0.13, \beta=0.12$, respectively). EDSS, physical comorbidity, depression, and anxiety all exhibited statistically significant indirect effects, in addition to statistically significant direct effects, on HRQoL (table 1). With both direct and indirect effects considered, EDSS had the strongest adverse effect on HRQoL ( $\beta=-0.52$ ), closely followed by depression

\section{Figure 2 Recruitment process and flow of participants in multiple sclerosis (MS) clinics}

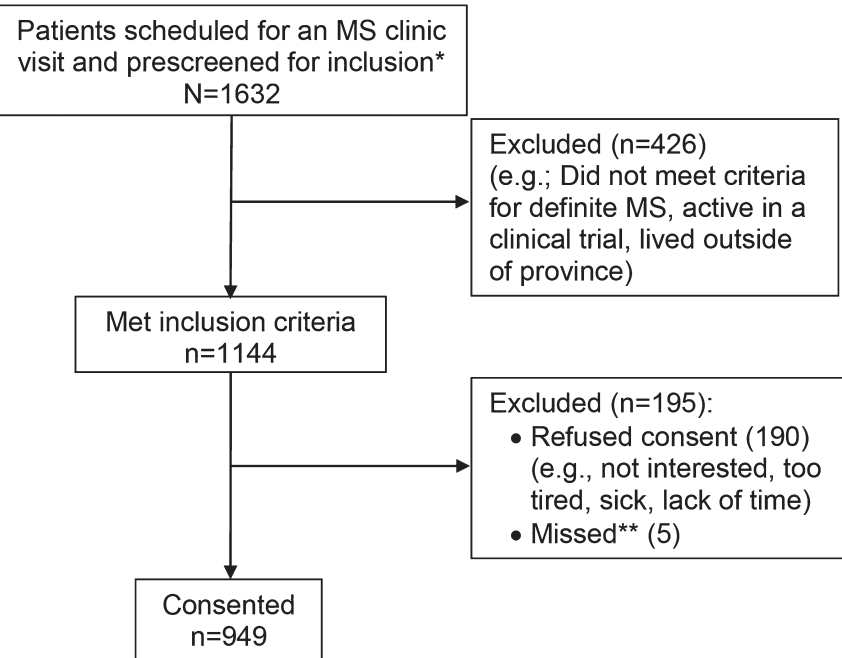

\footnotetext{
*Patients seen multiple times (e.g., for relapse visits or clinical trials) within the recruitment period were only counted once. ${ }^{*}$ Recruiter missed participants in the clinic. Thus, participation rate $=949 /(1,144-5)=82.6 \%$.
}

symptoms $(\beta=-0.50)$, then anxiety symptoms $(\beta=$ $-0.34)$, fatigue $(\beta=-0.27)$, and physical comorbidity $(\beta=-0.20)$. The influence of EDSS on HRQoL was primarily direct while the influence of depression symptoms on HRQoL was more equally split between direct and indirect paths. For anxiety symptoms and physical comorbidity, the proportion of the overall association with HRQoL arising from indirect effects was larger than that from direct effects. The predictors together accounted for $63 \%$ of the variance in the HRQoL variable.

DISCUSSION We investigated direct and indirect effects of disability, depression, anxiety, fatigue, and physical comorbidity on HRQoL in MS. The effect of physical comorbidity on HRQoL in MS has rarely been considered. One study restricted to relapsingremitting MS $(n=262)$ that used the Short Form36 health survey reported poorer physical HRQoL in patients with comorbid musculoskeletal and respiratory conditions compared to patients without those disorders. This study also found poorer mental HRQoL in patients with headaches and urinary and digestive tract problems. ${ }^{24}$ Of 335 people with MS who responded to the Canadian Community Health Survey, those with one or more comorbid illnesses had a lower mean HRQoL as measured by the HUI3 than those without a comorbidity (0.52 vs 0.64). ${ }^{25}$ Using the Short Form-12, the association of comorbidity and HRQoL was investigated with 8,983 participants in the North American Research Committee on Multiple Sclerosis (NARCOMS) registry. ${ }^{26}$ After adjustment for sociodemographic and clinical factors, physical HRQOL decreased as the number of comorbidities increased. In addition, participants with any mental comorbidity had worse mental HRQoL than those without a mental comorbidity. By contrast, one study that examined only comorbid migraine reported no effect on physical or mental HRQoL composite scores from the MSQoL-54. ${ }^{27}$ However, the sample size of this study was small (44 patients with and 44 without comorbid migraine) and the analyses may have been underpowered. Furthermore, while the MSQoL-54 composite scores were unaffected, patients with comorbid migraine had worse scores on subscales for role limitations due to physical problems, bodily pain, and health perceptions. ${ }^{27}$

Our results are consistent with this limited available research in demonstrating that physical comorbidity diminishes HRQoL in MS. The relation of physical comorbidity to HRQoL was weaker in comparison to other predictors examined and modification of factors such as disability and depression would clearly be expected to result in greater improvements in HRQoL. Nonetheless, the unstandardized 


\begin{tabular}{|c|c|c|}
\hline Table 2 & \multicolumn{2}{|c|}{$\begin{array}{l}\text { Characteristics of the multiple sclerosis } \\
\text { sample }\end{array}$} \\
\hline \multicolumn{2}{|l|}{ Variables } & Values \\
\hline \multicolumn{3}{|l|}{ Sex, n (\%) } \\
\hline \multicolumn{2}{|l|}{ Female } & 714 (75.2) \\
\hline \multicolumn{2}{|l|}{ Male } & 235 (24.8) \\
\hline \multicolumn{2}{|c|}{ Age, $y$, mean (SD) } & 48.62 (11.42) \\
\hline \multicolumn{2}{|c|}{ Disease duration, $y$, mean (SD) } & $15.43(10.21)$ \\
\hline \multicolumn{2}{|c|}{ EDSS, median (IQR) } & $2.50(3.50)$ \\
\hline \multicolumn{3}{|c|}{ Clinical course, $n(\%)$} \\
\hline \multicolumn{2}{|l|}{ RR } & $687(72.4)$ \\
\hline \multicolumn{2}{|l|}{ SP } & $193(20.3)$ \\
\hline \multicolumn{2}{|l|}{ PP } & $60(6.3)$ \\
\hline \multicolumn{2}{|l|}{ CIS } & $5(0.5)$ \\
\hline \multicolumn{2}{|c|}{ Unknown } & $3(0.3)$ \\
\hline \multicolumn{3}{|c|}{ Physical comorbidities, n (\%) } \\
\hline \multicolumn{2}{|l|}{0} & $405(42.7)$ \\
\hline \multicolumn{2}{|l|}{1} & $279(29.4)$ \\
\hline \multicolumn{2}{|l|}{2} & $149(15.7)$ \\
\hline \multicolumn{2}{|l|}{3} & $76(8.0)$ \\
\hline \multicolumn{2}{|l|}{$\geq 4$} & $40(4.2)$ \\
\hline \multicolumn{3}{|c|}{ Current DMT use, n (\%) } \\
\hline \multicolumn{2}{|l|}{ No } & 477 (50.3) \\
\hline \multicolumn{2}{|l|}{ Yes } & $466(49.1)$ \\
\hline \multicolumn{2}{|c|}{ DFIS, mean (SD) } & 12.55 (8.25) \\
\hline \multicolumn{2}{|c|}{ HADS-D, mean (SD) } & 4.72 (3.58) \\
\hline \multicolumn{2}{|c|}{ HADS-A, mean (SD) } & $6.56(4.06)$ \\
\hline \multicolumn{2}{|c|}{ HUI3 global index, mean (SD) } & $0.54(0.32)$ \\
\hline
\end{tabular}

Abbreviations: $\mathrm{CIS}=$ clinically isolated syndrome; DFIS = Daily Fatigue Impact Scale; DMT = disease-modifying therapy; EDSS = Expanded Disability Status Scale; Hospital Anxiety and Depression Scale-anxiety; HADS-D = Hospital Anxiety and Depression Scale-depression; HUI3 = Health Utilities Index Mark 3; IQR = interquartile range; $\mathrm{PP}=$ primary progressive; $\mathrm{RR}=$ relapsing-remitting; $\mathrm{SP}=$ secondary progressive.

regression coefficient corresponding to the total effect of physical comorbidity on HUI3 was -0.05 , indicating that an increase of one physical comorbidity corresponded to a decrease of 0.05 points on the HUI3 global utility score. As differences of 0.03 or greater in HUI3 global utility scores are considered clinically important ${ }^{28}$ our findings suggest that screening and treatment for physical comorbidities could lead to meaningful improvement in HRQoL for patients with MS and demonstrate the importance of an awareness of the potential effects of physical comorbidity in MS clinical practice. However, because our study did not evaluate interventions for comorbidities, future research is necessary to determine if such interventions can improve HRQoL for those with MS.
In addition to contributing to the emerging evidence that HRQoL is worse for patients with MS with physical comorbidities, the present findings also offer new information about the mechanisms of the effect. Our results suggest that, although the direct influence on HRQoL is modest, physical comorbidity worsens symptoms of depression, anxiety, and fatigue, all of which are themselves important determinants of HRQoL for persons with MS. Past research reporting associations of physical comorbidity with depression and fatigue provides support for the intermediate relations we observed. For depression, in 8,983 participants from the NARCOMS registry, those reporting the presence of any physical comorbidity had an increased risk of a diagnosis of depression after MS onset and were more likely to self-report current depressive symptoms that suggested probable major depression. ${ }^{29}$ For fatigue, in a study of the effectiveness of fatigue self-management education in 181 patients with MS, those reporting arthritis, diabetes, heart conditions, and respiratory conditions had worse fatigue at baseline, while comorbid diabetes and arthritis moderated fatigue improvement over the course of the trial..$^{30}$ Thus, although few studies have explored the important intermediate relations that we observed, support for such relations does exist.

The effect of comorbid psychiatric conditions on HRQoL in MS has received more attention than the effect of physical comorbidities. Specifically, worse HRQoL has been observed for patients with MS with clinical diagnoses of depression and selfreported depression symptoms in both crosssectional and longitudinal studies. ${ }^{1,26,31-33}$ As in the present study, when multiple determinants of HRQoL in MS have been examined concurrently, depression has frequently emerged as one of the most, if not the most, important predictors. ${ }^{1}$ While disability status was most strongly associated with HRQoL in our study, the magnitude of the overall relation between depressive symptoms and HRQoL was similar ( $\beta=-0.52$ for EDSS vs $\beta=-0.50$ for depression). Importantly, however, our results suggest that the relation of depression to HRQoL is almost equally split between a direct association and an indirect association through its influence on anxiety or fatigue. Fatigue is one of the most frequent and disabling symptoms of $\mathrm{MS}^{34}$ and it often emerges as an important predictor of HRQoL. ${ }^{1}$ An association between depression and fatigue is well-established ${ }^{34}$ and it is not surprising that overlap between these factors in their relation to HRQoL exists. Thus, our results indicate the influence of depression on HRQoL in MS may be partly mediated by its effects on fatigue, although it could be argued that the disabling nature of fatigue may contribute to depression, as opposed to depression influencing fatigue in MS. 


\begin{tabular}{|ll|}
\hline Table 3 & $\begin{array}{l}\text { Frequency of comorbid conditions in the } \\
\text { multiple sclerosis sample }\end{array}$ \\
\hline Condition & No. (\%) \\
\hline Depression & $274(29.0)$ \\
\hline Hypertension & $168(17.8)$ \\
\hline Migraine & $164(17.3)$ \\
\hline Hypercholesterolemia & $118(12.4)$ \\
\hline Anxiety disorder & $109(11.5)$ \\
\hline Lung disease (COPD) & $93(9.8)$ \\
\hline Irritable bowel syndrome & $75(7.9)$ \\
\hline Thyroid disease (not cancer) & $74(7.8)$ \\
\hline Osteoporosis & $57(6.0)$ \\
\hline Cataracts & $42(4.4)$ \\
\hline Diabetes mellitus & $38(4.0)$ \\
\hline Rheumatoid arthritis & $35(3.7)$ \\
\hline Fibromyalgia & $34(3.6)$ \\
\hline Heart disease & $28(3.0)$ \\
\hline Inflammatory bowel disease & $18(1.9)$ \\
\hline Glaucoma & $14(1.5)$ \\
\hline Peripheral vascular disease & $14(1.5)$ \\
\hline Bipolar disorder & $13(1.4)$ \\
\hline Epilepsy & $11(1.2)$ \\
\hline Schizus & $9(1.0)$ \\
\hline & $1(0.1)$ \\
\hline
\end{tabular}

Abbreviation: $\mathrm{COPD}=$ chronic obstructive pulmonary disease.

The current cross-sectional analysis does not allow us to investigate the directionality of the relation; this will require future longitudinal analyses.

In contrast to depression, the relation between anxiety and HRQoL in MS has been examined far less often. ${ }^{8,35,36}$ While prior studies generally report reduced HRQoL when anxiety is evaluated independently, ${ }^{8,37,38}$ this association tends to be weaker than that for depression, ${ }^{37,38}$ and anxiety has not always been a predictor of HRQoL once EDSS and depression were controlled for. ${ }^{8}$ In the current study, increased symptoms of anxiety were directly associated with reduced HRQoL and, as was the case for physical comorbidity, the relation between anxiety and HRQoL was stronger when its indirect influence via depression and fatigue was considered. Anxiety did not affect HRQoL as strongly as EDSS and depression, however. Of note, the HADS does not distinguish subtypes of anxiety disorders and likely identifies generalized anxiety and panic symptoms rather than symptoms of more situation-specific disorders such as social anxiety. Social anxiety was found to be prevalent and associated with lower HRQoL in MS. ${ }^{38}$ Thus, the association of anxiety with HRQoL may still be underestimated and future research is necessary to better understand the contribution of anxiety in its various forms to HRQoL in MS.

Limitations of the present study include its crosssectional nature and our inability to establish causal relations among the constructs examined. Physical comorbidity was measured using only self-reported data although the questionnaire used has been validated against medical record review in patients with $\mathrm{MS}^{14}$ and self-reported comorbidity has been associated with HRQoL at least as well as comorbidity data from medical records. ${ }^{39,40}$ The present analysis did not differentiate the relative importance of individual physical comorbidities and instead investigated the total burden of common physical comorbidities in MS. Individual physical comorbidities likely vary in their influence on $\mathrm{HRQOL}^{26}$ and further investigation is necessary to identify those contributing most to HRQoL, as well as the effects of the duration, severity, and treatment of the comorbidities. We recruited participants through MS clinics and included a high proportion of relapsing-onset cases. Thus, the ability to generalize findings to those with progressive-onset disease may be limited. The model did not include all possible contributors to HRQoL in MS; future investigations should attempt to evaluate others, such as cognition. Finally, the $\chi^{2}$ value for the model was significant, suggesting inadequate model fit. However, as noted previously, small departures from the sample covariances may reach statistical significance when large samples such as ours are examined. In contrast, CFI and RMSEA values were within the range indicating satisfactory fit.

The strengths of our study include the large sample and the focus on a clinically relevant and important knowledge gap in MS. Importantly, both the independent and overlapping contributions of disability, symptoms of depression and anxiety, fatigue, and physical comorbidity to HRQoL were examined, with demographic and disease-related variables accounted for. Neurologic disability, symptoms of depression and anxiety, fatigue, and physical comorbidity were all associated with HRQoL and, together, accounted for a large proportion of variance in HRQoL. While the direct associations of physical comorbidity and anxiety with HRQoL were relatively modest, their associations increased when their effects on depression and fatigue were considered. Interventions that reduce neurologic disability are expected to yield the most substantial improvement in HRQoL for those with MS. Nonetheless, as a complement to treatments aimed at reducing neurologic disability, interventions targeting other factors amenable to change, particularly depression but also anxiety, fatigue, and physical 
comorbidities, may all result in meaningful improvements in HRQoL, as well. Our findings point to the importance of further research confirming the efficacy of such interventions.

\section{AUTHOR CONTRIBUTIONS}

Lindsay Berrigan was responsible for analysis and interpretation of the data and drafting and revising the manuscript for intellectual content. John Fisk contributed to the conceptualization and design of the study, interpretation of the data, and revising the manuscript for intellectual content. Scott Patten contributed to the conceptualization and design of the study, interpretation of the data, and revising the manuscript for intellectual content. Helen Tremlett contributed to the conceptualization and design of the study, interpretation of the data, and revising the manuscript for intellectual content. Christina Wolfson contributed to the conceptualization and design of the study and revising the manuscrip for intellectual content. Sharon Warren contributed to the conceptualization and design of the study. Kirsten Fiest contributed to the interpretation of the data and revising the manuscript for intellectual content. Kyla McKay contributed to the interpretation of the data and revising the manuscript for intellectual content. Ruth Ann Marrie was responsible for the conceptualization and design of the study and contributed to analysis and interpretation of the data and revising the manuscript for intellectual content.

\section{ACKNOWLEDGMENT}

The authors thank the research participants who volunteered their time; the neurologists and staff in all 4 clinics who contributed to this study through patient examination and facilitated access to clinical data; and the research assistants who coordinated data collection (Anna-Marie Bueno, Stacey Tkachuk, Nicholas Hall, Karen Turpin, Leanne Bergsma, Amy Cuthbertson, Alexandra Nelson, Beth DeCoste) and facilitated data manipulation (Tom Duggan, Karen Stadnyk).

\section{STUDY FUNDING}

The study was sponsored by the Canadian Institutes of Health Research (CIHR: CBG 101829), the Rx \& D Health Research Foundation, by a Don Paty Career Development Award from the Multiple Sclerosis Society of Canada to R.A.M., by a Manitoba Research Chair from Research Manitoba to R.A.M., and by a postdoctoral fellowship from the MS Society of Canada to L.I.B.

\section{DISCLOSURE}

L. Berrigan has received research support from the Multiple Sclerosis Society of Canada, Postdoctoral Fellowship. J. Fisk is the Director of the endMS Atlantic Regional Research and Training Centre, which is funded by the MS Society of Canada and receives grant funding from CIHR and the National Multiple Sclerosis Society. S. Patten was a member of an advisory board for Servier, Canada. He has received honoraria for reviewing investigator-initiated grant applications submitted to Lundbeck and Pfizer and has received speaking honoraria from Teva and Lundbeck. He is the Editor-in-Chief for the Canadian Journal of Psychiatry and a member of the editorial board of Chronic Diseases and Injuries in Canada. He is the recipient of a salary support award (Senior Health Scholar) from Alberta Innovates: Health Solutions and receives research funding from CIHR, the Institute of Health Economics, and the Alberta Collaborative Research Grants Initiative. H. Tremlett is funded by the MS Society of Canada (Don Paty Career Development Award) and is a Michael Smith Foundation for Health Research Scholar and the Canada Research Chair for Neuroepidemiology and Multiple Sclerosis. She has received research support from the National MS Society, CIHR, and the UK MS Trust; and speaker honoraria and/or travel expenses to attend conferences from the Consortium of MS Centres (2013), the National MS Society (2012, 2014), Bayer Pharmaceuticals (2010), Teva Pharmaceuticals (2011), ECTRIMS (2011, 2012, 2013, 2014), UK MS Trust (2011), the Chesapeake Health Education Program, US Veterans Affairs (2012), Novartis Canada (2012), Biogen Idec (2014), and American Academy of Neurology (2013, 2014, 2015). Unless otherwise stated, all speaker honoraria are either donated to an MS charity or to an unrestricted grant for use by her research group. C. Wolfson receives research funding from the MS Society of Canada, CIHR, Canada Foundation for Innovation, and the National MS Society, and has received one speaking honorarium from Novartis. S. Warren receives research funding from CIHR. K. Fiest and K. McKay report no disclosures relevant to the manuscript. R. Marrie receives research funding from CIHR, Public Health Agency of Canada, Research Manitoba, Health Sciences Centre Foundation, MSSC, Multiple Sclerosis Scientific Foundation, $\mathrm{Rx} \& \mathrm{D}$ Health Research Foundation, and National Multiple Sclerosis Society, and has conducted clinical trials funded by sanofi-aventis. Go to Neurology.org for full disclosures.

Received May 27, 2015. Accepted in final form January 5, 2016.

\section{REFERENCES}

1. Papuc E, Stelmasiak Z. Factors predicting quality of life in a group of Polish subjects with multiple sclerosis: accounting for functional state, socio-demographic and clinical factors. Clin Neurol Neurosurg 2012;114: 341-346.

2. Hermann B, Vickrey B, Hays RD, et al. A comparison of health-related quality of life in patients with epilepsy, diabetes and multiple sclerosis. Epilepsy Res 1996;25: 113-118.

3. Rudick RA, Miller DM, Clough JD, Gragg LA, Farmer RG. Quality of life in multiple sclerosis: comparison with inflammatory bowel disease and rheumatoid arthritis. Arch Neurol 1992;49:1237-1242.

4. Wu N, Minden SL, Hoaglin DC, Hadden L, Frankel D. Quality of life in people with multiple sclerosis: data from the Sonya Slifka Longitudinal Multiple Sclerosis Study. J Health Hum Serv Adm 2007;30:233-267.

5. Marrie RA, Cohen J, Stuve O, et al. A systematic review of the incidence and prevalence of comorbidity in multiple sclerosis: overview. Mult Scler 2015;21:263-281.

6. Gijsen R, Hoeymans N, Schellevis FG, Ruwaard D, Satariano WA, van den Bos GA. Causes and consequences of comorbidity: a review. J Clin Epidemiol 2001;54: 661-674.

7. Janardhan V, Bakshi R. Quality of life in patients with multiple sclerosis: the impact of fatigue and depression. J Neurol Sci 2002;205:51-58.

8. Janssens ACJW, Van Doorn PA, De Boer JB, et al. Anxiety and depression influence the relation between disability status and quality of life in multiple sclerosis. Mult Scler 2003;9:397-403.

9. Poser CM, Paty DW, Scheinberg L, et al. New diagnostic criteria for multiple sclerosis: guidelines for research protocols. Ann Neurol 1983;13:227-231.

10. Polman CH, Reingold SC, Edan G, et al. Diagnostic criteria for multiple sclerosis: 2005 revisions to the "McDonald Criteria." Ann Neurol 2005;58:840-846.

11. Kurtzke JF. Rating neurologic impairment in multiple sclerosis: an expanded disability status scale (EDSS). Neurology 1983;33:1444-1452.

12. Feeny DH, Furlong W, Torrance GW, et al. Multiattribute and single-attribute utility functions for the Health Utilities Index Mark 3 system. Med Care 2002;40: 113-128.

13. Fisk JD, Brown MG, Sketris IS, Metz LM, Murray TJ, Stadnyk KJ. A comparison of health utility measures for the evaluation of multiple sclerosis treatments. J Neurol Neurosurg Psychiatry 2005;76:58-63.

14. Horton M, Rudick RA, Hara-Cleaver C, Marrie RA. Validation of a self-report comorbidity questionnaire for multiple sclerosis. Neuroepidemiology 2010;35:83-90. 
15. Zigmond AS, Snaith RP. The Hospital Anxiety and Depression Scale. Acta Psychiatr Scand 1983;67:361-370.

16. Honarmand K, Feinstein A. Validation of the Hospital Anxiety and Depression Scale for use with multiple sclerosis patients. Mult Scler 2009;15:1518-1524.

17. Fisk JD, Doble SE. Construction and validation of a fatigue impact scale for daily administration (D-FIS). Qual Life Res 2002;11:263.

18. Arbuckle JL. AMOS 20 User's Guide. Chicago: SPSS; 2011.

19. Byrne BM. Structural Equation Modeling With AMOS: Basic Concepts, Applications, and Programming. Mahwah, NJ: Lawrence Erlbaum Associates; 2001.

20. Tabachnick BG, Fidell LS. Using Multivariate Statistics, 5th ed. New York: Pearson Education; 2007.

21. Moffitt TE, Harrington H, Caspi A, et al. Depression and generalized anxiety disorder: cumulative and sequential comorbidity in a birth cohort followed prospectively to age 32 years. Arch Gen Psychiatry 2007;64:651-660.

22. Kline RB. Principles and Practices of Structural Equation Modeling, 2nd ed. New York: The Guilford Press; 2005.

23. Feng Y, Bernier J, McIntosh C, Orpana H. Validation of disability categories derived from Health Utilities Index Mark 3 scores. Health Rep 2009;20:43-50.

24. Turpin KV, Carroll LJ, Cassidy JD, Hader WJ. Deterioration in the health-related quality of life of persons with multiple sclerosis: the possible warning signs. Mult Scler 2007;13:1038-1045.

25. Warren SA, Turpin KV, Pohar SI, Jones CA, Warren KG. Comorbidity and health-related quality of life in people with multiple sclerosis. Int J MS Care 2009;11:6-16.

26. Marrie RA, Horwitz R, Cutter G, Tyry T. Cumulative impact of comorbidity on quality of life in MS. Acta Neurol Scand 2012:125;180-186.

27. Villani V, Prosperini L, Pozzilli C, Salvetti M, Sette G. Quality of life of multiple sclerosis patients with comorbid migraine. Neurol Sci 2011;32(suppl 1):S149-S151.

28. Horsman J, Fulong W, Feeny D, Torrance G. The Health Utilities Index (HUI): concepts, measurement properties and applications. Health Qual Life Outcomes 2003;1:54.

29. Marrie RA, Cutter G, Tyry T, Campagnolo D, Vollmer T. Effect of physical comorbidities on risk of depression in multiple sclerosis. Int J MS Care 2009;11:161-165.
30. Finlayson M, Preissner K, Cho C. Impact of comorbidity on fatigue management intervention outcomes among people with multiple sclerosis. Int J MS Care 2013;15: 21-26.

31. Carta MG, Moro MF, Lorefice L, et al. Multiple sclerosis and bipolar disorders: the burden of comorbidity and its consequences on quality of life. J Affective Disord 2014; 167:192-197.

32. Goldman Consensus Group. The Goldman Consensus statement on depression in multiple sclerosis. Mult Scler 2005;11:328-337.

33. Tepavcevic DK, Pekmezovic T, Stojsavljevic N, et al. Change in quality of life and predictors of change among patients with multiple sclerosis: a prospective cohort study. Qual Life Res 2014;23:1027-1037.

34. Penner IK, Bechtel N, Raselli C, et al. Fatigue in multiple sclerosis: relation to depression, physical impairment, personality and action control. Mult Scler 2007;13: 1161-1167.

35. Dubayova T, Krokavcova M, Nagyova I, et al. Type D, anxiety and depression in association with quality of life in patients with Parkinson's disease and patients with multiple sclerosis. Qual Life Rese 2013;22:1353-1360.

36. Szilasiova J, Krokavcova M, Gdovinova Z, Rosenberger J, Van Dijk JP. Quality of life in patients with multiple sclerosis in Eastern Slovakia. Disabil Rehabil 2011;33: 1587-1593.

37. Motl RW, McAuley E, Snook EM, Gliottoni RC. Physical activity and quality of life in multiple sclerosis: intermediary roles of disability fatigue, mood, pain, selfefficacy and social support. Psychol Health Med 2009; 14:111-124.

38. Poder K, Ghatavi K, Fisk JD, et al. Social anxiety in a multiple sclerosis clinic population. Mult Scler 2009;15: 393-398.

39. Bayliss EA, Ellis JL, Steiner JF. Subjective assessments of comorbidity correlate with quality of life health outcomes: initial validation of a comorbidity assessment instrument. Health Qual Life Outcomes 2005;2:51.

40. Voaklander D, Kelly KD, Jones CA, Suarez-Almazor ME. Self report co-morbidity and health related quality of life: a comparison with record based co-morbidity measures. Soc Indic Res 2004;66:213-228.

\section{Save These Dates for AAN CME Opportunities!}

Mark these dates on your calendar for exciting continuing education conferences by the American Academy of Neurology. Learn more at $A A N$.com/conferences.

\section{AAN Annual Meeting}

- April 15-21, 2016, Vancouver, BC, Canada, Vancouver Convention Centre 